Laser Chem., 1999, Vol. 19, pp. 149-152

Reprints available directly from the publisher Photocopying permitted by license only
(C) 1999 OPA (Overseas Publishers Association) N.V. Published by license under the Harwood Academic Publishers imprint, part of The Gordon and Breach Publishing Group.

\title{
FEMTOSECOND MATERIAL RESPONSE PROBED BY PHASE-STABILIZED OPTICAL HETERODYNE DETECTED IMPULSIVE STIMULATED RAMAN SCATTERING
}

\author{
TAHEI TAHARA* and SHIGEKI MATSUO \\ Institute for Molecular Science, Myodaiji, Okazaki 444, Japan
}

(Received 7 April 1997)

Femtosecond material response of several liquid samples were measured with use of a newly developed "phase-stabilized" optical heterodyne detected impulsive stimulated Raman scattering (ISRS) spectrometer. In this apparatus, the ISRS signal generated with ordinary transient grating geometry is mixed with femtosecond optical pulses (local oscillator, LO) in an interferometer, and the interfered intensity is detected. The subwavelength-accuracy adjustment/stabilization is achieved for the optical path length in the interferometer so that the relative optical phase between the ISRS signal and LO can be controlled. The ISRS signals linearized to each tensor element of the third-order response function were obtained, and discussed.

Keywords: Femtosecond spectroscopy; Raman spectroscopy; time-domain spectroscopy; optical heterodyne detection; ultrafast dynamics; instrumentation

Thanks to recent advances of the ultrafast laser technology, we are now able to observe femtosecond material response directly in the time domain. For the measurement of Raman-active responses, impulsive stimulated Raman scattering (ISRS) spectroscopy and optical heterodyne detected Raman-induced Kerr effect spectroscopy (OHD-RIKES) have been widely utilized. Although both these time-domain spectroscopies give information about the third-order nonlinear response function, each has its advantages over the other; ISRS spectroscopy is

\footnotetext{
${ }^{*}$ Corresponding author. Tel.: +81-564-55-7391, Fax: $+81-564-54-2254$, e-mail: tahara@ims.ac.jp
} 
applicable to the measurements for each tensor element of the response function, while OHD-RIKES gives signals linearized to the material response. As a method having advantages of both ISRS spectroscopy and OHD-RIKES, optical heterodyne detected impulsive stimulated Raman scattering (OHD-ISRS) spectroscopy has been proposed and demonstrated [1-3]. Especially, we recently reported the OHD-ISRS measurements in which both complete heterodyning and the optical phase control were attained for the first time [3].

Figure 1 shows schematic diagram of OHD-ISRS measurements. The two pump pulses are focused into the sample with a certain crossing angle to generate transient grating. The material response is detected through the temporal change of the grating amplitude, which is monitored by diffraction of the probe pulse (impulsive stimulated Raman scattering, ISRS). The generated ISRS beam is mixed with the probe pulse which serves as local oscillator (LO), and then the interfered intensity is detected. The obtained OHD-ISRS signal intensity depends on the relative optical phase between the ISRS signal and LO (heterodyning phase, $\psi$ ) as follows,

$$
\operatorname{SOHD-ISRS}_{\mathrm{O}}(\tau) \propto \cos \psi \cdot \int_{-\infty}^{\infty} \chi(t) G(t-\tau) d t,
$$

where $\chi(t)$ is the material response function, and $G(t)$ is the intensity autocorrelation function of the input laser pulses. Therefore it is

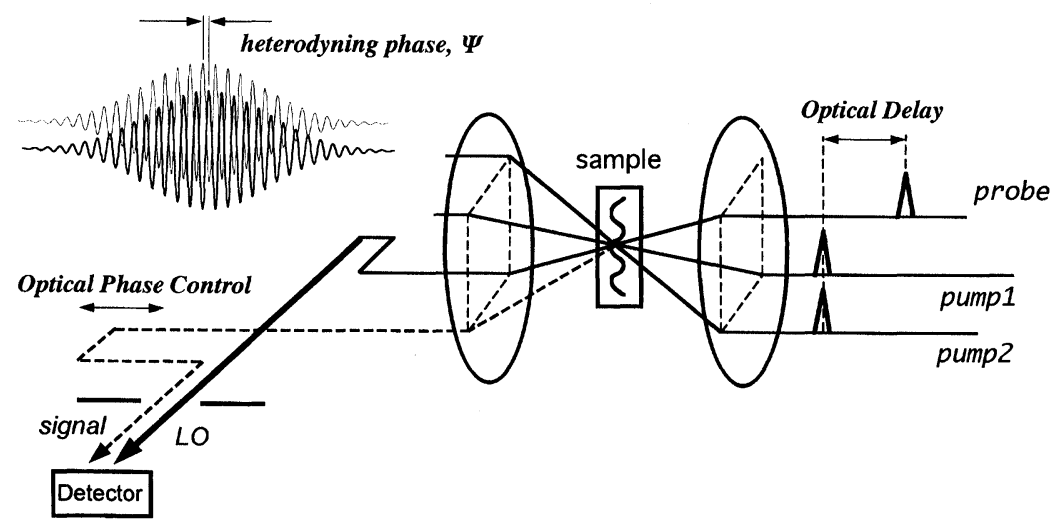

FIGURE 1 Schematic diagram of the OHD-ISRS measurement. 
necessary to control the relative optical phase between the ISRS signal and LO during measurements. For this purpose, optical path is actively adjusted while monitoring the interference of a reference laser, so that subwavelength-accuracy adjustment/stabilization is achieved for the optical path length of the interferometer. The maximum signal with the best signal-to-noise ratio is obtained in the measurements at the heterodyning phase of $\psi=0$.

Figure 2 shows the OHD-ISRS signals obtained from $\mathrm{CCl}_{4}$ with two polarization conditions which are sensitive to $\chi_{1111}$ and $\chi_{1122}$, respectively. In these data, beating nuclear response due to intramolecular vibrations of $\mathrm{CCl}_{4}$ is clearly seen, in addition to instantaneous electronic response found at the time origin. The phase of the beat is

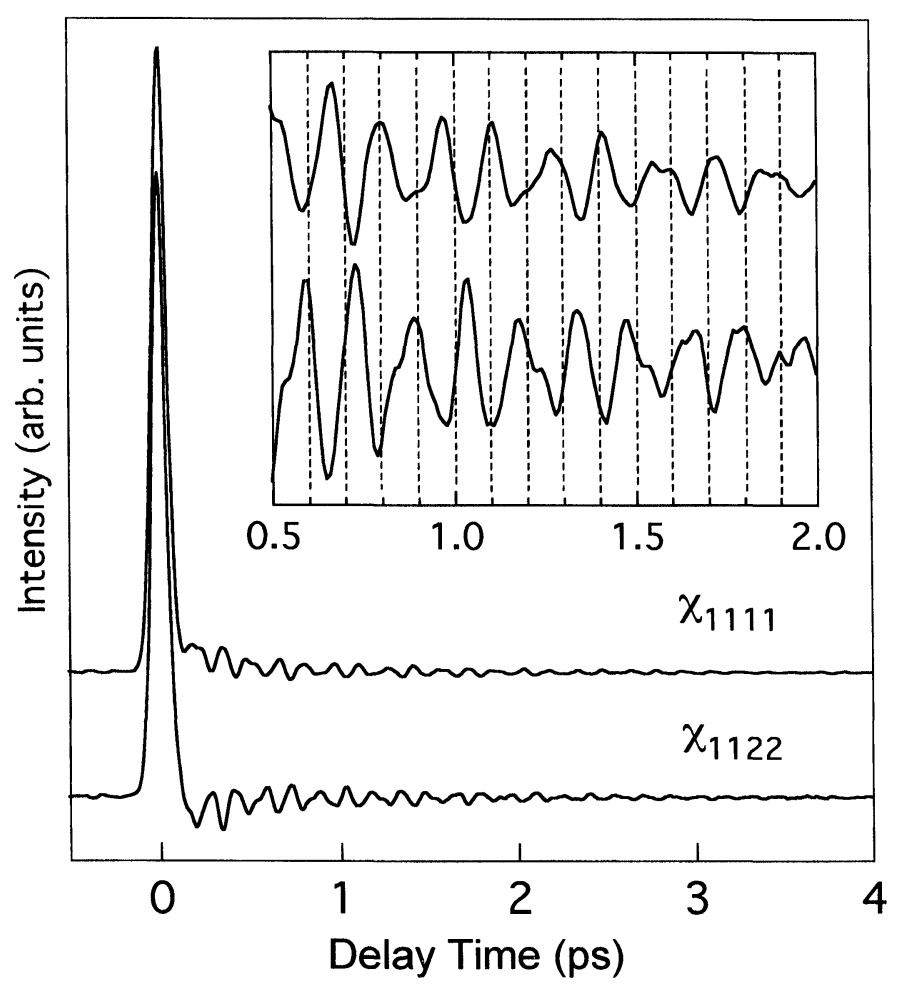

FIGURE 2 The OHD-ISRS signals obtained from carbon tetrachloride with two polarization conditions which are sensitive to $\chi_{1111}^{(3)}$ and $\chi_{1122}^{(3)}$. Inset: Expanded (upper: $\chi_{1111}^{(3)}$; lower: $\chi_{1122}^{(3)}$ ). 
different (almost opposite) between the $\chi_{1111}^{(3)}$ and $\chi_{1122}^{(3)}$ data. This phase change of the beat reflects the symmetry of the corresponding vibrational modes of $\mathrm{CCl}_{4}$.

The ratio between $\chi_{1111}^{(3)}$ and $\chi_{1122}^{(3)}$ can be related to Raman depolarization ratio $\rho$ as follows,

$$
\chi_{1122}^{(3)} \chi_{1111}^{(3)}=1-2 \rho .
$$

This formula implies that the anisotropic response corresponding to the $\rho$ value larger than 0.5 gives negative signal in the $\chi_{1122}^{(3)}$ measurement. The observed beats are predominantly due to two low-frequency non-totally symmetric vibrations of $\mathrm{CCl}_{4} \quad\left(\nu_{2}\right.$, $218 \mathrm{~cm}^{-1}$, E symmetry; $\nu_{4}, 314 \mathrm{~cm}^{-1}, \mathrm{~F}_{2}$ symmetry) whose $\rho$ value is 0.75 . It is noteworthy that the Fourier analysis shows that the beat also contains small contribution from the totally symmetric vibration $\left(\nu_{1}, 458 \mathrm{~cm}^{-1}, \mathrm{~A}_{1}, \rho=0\right)$, which does not change its sign. As manifested by this example, OHD-ISRS spectroscopy can afford unique data about the third-order nonlinear material response, which is not obtainable by either ordinary homodyne detected ISRS spectroscopy or OHD-RIKES.

\section{References}

[1] Vöringer, P. and Scherer, N. F. (1995). J. Phys. Chem., 99, 2684.

[2] Chang, Y. J., Cong, P. and Simon, J. D. (1995). J. Phys. Chem., 99, 7857.

[3] Matsuo, S. and Tahara, T. (1997). Chem. Phys. Lett., 264, 636. 